\title{
Overcoming obstacles: collaboration for change
}

\author{
M M Funnell \\ Michigan Diabetes Research and Training Centre, University of Michigan, 1331 E. Ann, Box 0580, Room 5111, Ann Arbor, \\ Michigan 48109-0580, USA \\ (Correspondence should be addressed to M M Funnell; Email: mfunnell@umich.edu)
}

\begin{abstract}
Effective diabetes care requires a partnership between prepared, proactive practice teams and informed, activated patients. Diabetes education helps to overcome many of the barriers to effective self-management by enabling people with diabetes to make informed decisions about their day-today self-care. Both psychosocial and health outcomes have been improved through a variety of training programmes; however, education must be coupled with ongoing self-management support if these benefits are to be sustained. The principal goal of diabetes education has undergone a major shift over the past few years - evolving from primarily didactic interventions, focused on encouraging patients to adhere to the prescribed therapy, towards more interactive learning that supports people in making informed, self-directed decisions.
\end{abstract}

European Journal of Endocrinology 151 T19-T22

It has become apparent that we need to reframe the patient-care-giver relationship to reflect the fact that people with diabetes are central to their own care. Care-givers need to understand their patients' goals and objectives for therapy so that both parties work towards the same goals. Patients also need to be informed about recommended metabolic and other parameters of quality diabetes care. Diabetes outcomes can then be improved through collaboration to identify barriers and to develop informed, individual and achievable treatment targets and self-care plans.

\section{Problems facing diabetes care}

Over the past two decades, new medications and technologies, together with a greater awareness of the psychological needs of people with diabetes, have facilitated significant advances in diabetes care. However, both healthcare professionals and patients continue to face difficulties in managing diabetes on a day-to-day basis. Many healthcare professionals are frustrated and dissatisfied by obstacles that limit their ability to care for their patients. Medical care systems are often inadequate and individuals may be unable to carry out or afford necessary self-care. As the global burden of diabetes increases, the challenge to achieve the best possible standard of care will become even greater.

As discussed in the previous paper (1), the practical and emotional burden of diabetes care lies primarily with patients. They are often expected to manage multiple metabolic parameters, including blood glucose levels, lipids and blood pressure, by making significant lifestyle changes and using complex treatment regimens.
Therapeutic issues, such as polypharmacy and the need for multiple insulin injections, contribute further to the daily burden of living with diabetes. Among all the therapies, technologies and pressures on care-givers to see more patients in ever shorter periods of time, people with diabetes may begin to feel that they are unimportant - that they are just a blood sugar number.

\section{The evidence base for education}

As defined in the Chronic Care Model developed by Wagner et al. (2), effective diabetes care requires a partnership between prepared, proactive practice teams and informed, activated patients (2). Two main areas need to be addressed if we are to help our patients become informed and involved in their own care. First, we must ensure that we deliver the best possible information in an effective manner. Secondly, information must be coupled with ongoing self-management support in order to sustain the type of behavioural changes required by people with a chronic illness.

The results of numerous studies of diabetes education have recently been summarised by several meta-analyses. These show that diabetes self-management education can improve both psychosocial and health (metabolic and clinical) outcomes (3-6). Unfortunately, there is no universally effective education programme and we must use the insights gained from these studies to develop tailored programmes taking into consideration each individual, their culture, the location in which training is delivered and the resources that are available. Studies indicate that training programmes incorporating behavioural and affective 
components are generally more effective than those that are strictly didactic or knowledge and skills based (7-9). We also know that group education may work better than individual education, as well as being more costeffective and efficient with regards to staff time $(6,10$, 11). Furthermore, education programmes that are tailored for the cultures and communities in which they are delivered are more effective than non-tailored programmes $(6,12)$. Although peer-led diabetes education groups can be very efficacious (6), some studies indicate that training programmes that include physicians, nurses and dieticians can have a greater impact (e.g. lower $\mathrm{HbA}_{1 \mathrm{c}}$ levels more effectively) than programmes that do not (13).

In recent years, diabetes education has evolved from primarily didactic interventions, focused on the acquisition of knowledge, towards more theoretical, interactive interventions based on patient empowerment (4). Whereas the aim of diabetes education used to be to get patients to adhere to treatment recommendations, we have learnt that its real value lies in providing information and support so that individuals with diabetes can make informed, self-directed decisions and manage their diabetes in a way that works for their health and their lives. Although knowledge continues to form an essential part of diabetes care, knowledge alone is not sufficient for the sustained behavioural changes that are necessary to manage diabetes for life (14).

\section{Collaborative care}

Effective long-term diabetes management requires 'collaborative care' in which people with diabetes receive ongoing support from care-givers across various aspects of self-care including education, goal-setting and behaviour change (15). Collaborating to develop informed, individual and achievable targets is an important part of helping patients achieve their goals.

A variety of ways of incorporating collaborative care into practical, cost-efficient interventions are currently being investigated. In particular, strategies are being developed to reduce the demand on physician time, which is both limited and expensive. Case managers and nurse-led clinics have been used successfully to improve outcomes in the USA and the UK respectively (16-20). In addition, information technologies ranging from simple telephone systems to sophisticated computer programs can be used to supplement care (15). In a study by Piette et al. (15), participants who received standard care plus bi-weekly automated telephone disease management calls with telephone follow-up by a nurse educator, reported fewer symptoms of depression $(P=0.023)$, greater self-efficacy to conduct self-care activities $(P=0.006)$ and fewer days in bed because of illness $(P=0.026)$ compared with those who received standard care alone.

Cost-effective ongoing collaborative care requires a team of people with different expertise, led by the person with diabetes. We need to consider the best ways of organising these teams, ensuring interaction between team members and assigning roles and responsibilities. Glasgow et al. (21) found that the most effective teams incorporated ongoing patientcentred education, goal-setting and collaboration into everyday care. Although there were key individuals responsible, everyone concerned with the provision of diabetes healthcare - physicians, nurses, dieticians and clerical staff - took responsibility for talking to patients about their goals and goal attainment.

Conveying the importance of diabetes self-management to patients is essential for collaborative care. Although people with diabetes are responsible for managing their condition on a day-to-day basis, we do not always make it clear that this has a huge bearing, not only on their lives today, but also on the prevention of complications that may damage their long-term health and quality of life.

We also need to reframe patient-care-giver relationships and interactions. As healthcare professionals, we offer expertise in diabetes while people with diabetes offer expertise on their own lives and priorities. It can be very effective to start a consultation by asking questions and listening to the response. Rather than increasing the consultation time, questions such as 'What would you like to accomplish today?' and 'What are your concerns?' can focus the visit and save time $(22,23)$. This also helps to prevent patients from raising important issues at the end of the consultation, thereby causing the session to overrun. Moreover, addressing the patients' concerns before those of the consultant reinforces a collaborative relationship and demonstrates that the patients' issues matter.

As part of the collaborative process, people with diabetes need to be informed about both the positive and negative aspects of diabetes management in order to make decisions about their self-care. However, while offering the best-known standard of care, healthcare professionals must consider and accommodate each individuals' choices. Understanding personal goals is critical for the successful long-term implementation of management strategies and to ensure that we offer the needed support.

The messages that people with diabetes receive from healthcare professionals can be very powerful. Caregivers tend to focus on glycated haemoglobin $\left(\mathrm{HbA}_{1 \mathrm{c}}\right)$ levels and other risk factors that are important from a clinical perspective but often overlook what is really important to patients - leading a normal life despite diabetes, and being able to manage diabetes in a way that gives flexibility, freedom and quality of life. This is especially true with respect to insulin therapy in patients with type 2 diabetes. Insulin is often used as a threat to encourage diet, exercise and the use of oral antidiabetic agents. However, fear tactics are generally ineffective and may contribute to negative behaviour. Using insulin as a threat may increase the 
patient's resistance to insulin in the future. It is more beneficial to ascertain each individuals' goals and fears and to then offer information and advice that will assist patients to achieve those goals.

There are many effective and practical ways to provide collaborative care. For example, a diabetes passport study is ongoing in Holland, in which people with diabetes carry a record of their goals and information to remind them, and their healthcare providers, when to screen for complications (24). The rationale for the diabetes passport is that treatment goals are more likely to be achieved if patients and care-givers are working towards the same goals. The Dose Adjustment For Normal Eating (DAFNE) trial (25) was based on a German study in which patients with type 1 diabetes were taught to control their glucose levels by adjusting insulin injections, rather than diet and lifestyle (26). DAFNE showed that patients undergoing a similar selfmanagement training course in the UK had significantly reduced glycated haemoglobin levels, and dramatically improved quality of life outcomes. In another example, a problem-based, culturally specific programme was initiated for African-Americans living in inner-city Detroit (27). The curriculum for this successful group programme was provided based on the participants' questions, experiences, and goals.

\section{Conclusions}

As healthcare professionals, we need to collaborate with our patients to develop individual action plans that incorporate provision for ongoing assessment of self-management beliefs, behaviour and knowledge, the identification of barriers and supports to goal attainment, personal goals, and individually tailored strategies and problem-solving ideas (Fig. 1). Asking questions and listening to the responses is a critical part of developing a collaborative relationship and the goal-setting process. Information needs to be framed to meet patients' goals and needs. Both diabetes care and effective self-care education must be tailored for the individual, taking into consideration culture, age, health status and personal preferences.

Ongoing support is crucial if people with diabetes are to sustain the long-term behavioural changes needed to achieve their treatment goals. Because it is ultimately the responsibility of patients to take charge of their diabetes management, it is vital that they are provided with the knowledge they need to make informed decisions about their own health. As healthcare professionals, our role is to collaborate with our patients, providing the necessary knowledge and skills, supporting their self-selected behavioural changes, and assisting them to discover their own innate ability to gain mastery over their diabetes.

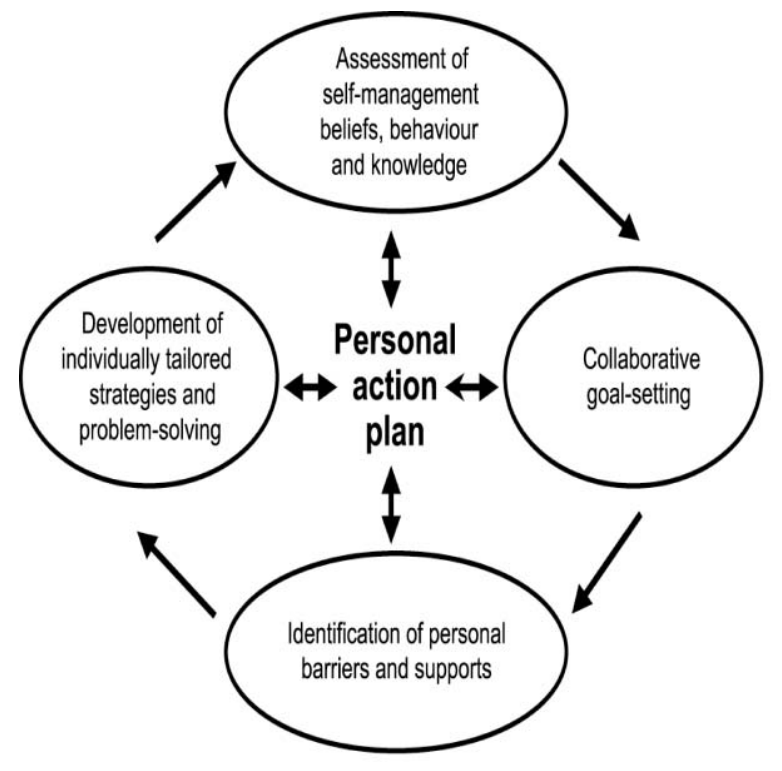

Figure 1 An integrated training model for successful self-management of chronic illnesses. (Adapted from (20) with permission.)

\section{Acknowledgements}

Work on this article was supported in part by grant number NIH5P60 DK20572 and 1R18 OK062323-01 from the National Institute of Diabetes and Digestive and Kidney Diseases of the National Institutes of Health.

\section{References}

1 Skinner TC. Psychological barriers. European Journal of Endocrinology 2004151 (Suppl) S13-S17.

2 Wagner EH. The role of patient care teams in chronic disease management. British Medical Journal 2000320 569-572.

3 Brown SA. Interventions to promote diabetes self-management: state of the science. Diabetes Educator 199925 (Suppl) 52-61.

4 Norris SL, Engelgau MM \& Naranyan KMV. Effectiveness of selfmanagement training in type 2 diabetes: a systematic review of randomized controlled trials. Diabetes Care $200124561-587$.

5 Norris SL, Lau J, Smith SJ, Schmid CH \& Engelgau MM. Self-management education for adults with type 2 diabetes: a meta-analysis on the effect on glycemic control. Diabetes Care 200225 1159-1171.

6 Barlow J, Wright C, Sheasby J, Turner A \& Hainsworth J. Selfmanagement approaches for people with chronic conditions: a review. Patient Education and Counselling 200248 177-187.

7 Roter DL, Hall JA, Merisca R, Nordstrom B, Cretin D \& Svarstad B. Effectiveness of interventions to improve patient compliance: a meta-analysis. Medical Care 199836 1138-1161.

8 Barlo J, Wright C, Sheasby J, Turner A \& Hainsworth J. Self-management approaches for people with chronic conditions: a review. Patient Education and Counselling $2002 \mathbf{4 8} 177-187$.

9 Skinner TC, Cradock S, Arundel F \& Graham W. Lifestyle and behavior: four theories and a philosophy. Self-mangement education for individuals newly diagnosed with type 2 diabetes. Diabetes Spectrum $2003 \mathbf{1 6} 75-80$.

10 Norris SL. Self-management education in type 2 diabetes. Practical Diabetology 200322 7-13.

11 Sarkisian CA, Brown AF, Norris KC, Wintz RL \& Mangione CM. A systematic review of diabetes self-care interventions for older, 
African-American, or Latino adults. Diabetes Education 200329 $467-479$.

12 Brown SA, Harrist RB, Villagomez ET, Segura M, Barton SA \& Hanis CL. Gender and treatment differences in knowledge, health beliefs, and metabolic control in Mexican Americans with type 2 diabetes. Diabetes Educator 200026 425-438.

13 Gary TL, Genkinger JM, Guallar E, Peyrot M \& Brancati FL. Metaanalysis of randomized educational and behavioral interventions in type 2 diabetes. Diabetes Education 200329 488-501.

14 Piette JD \& Glasgow RE. Education and self-monitoring of blood glucose. In Evidence-based Diabetes Care, pp 207-251. Eds HC Gerstein \& RB Haynes. Ontario, CA: BC Decker Inc., 2001.

15 Piette JD, Weinberger M \& McPhee SJ. The effect of automated calls with telephone nurse follow-up on patient-centered outcomes of diabetes care: a randomized, controlled trial. Medical Care $200038218-230$.

16 Aubert RE, Herman WH, Waters J, Moore W, Sutton D, Peterson BL, Bailey CM \& Koplan JP. Nurse case management to improve glycemic control in diabetic patients in a health maintenance organization. A randomized, controlled trial. Annals of Internal Medicine 1998129 605-612.

17 Hiss RG, Gillard ML, Armbruster BA \& McClure LA. Comprehensive evaluation of community-based diabetic patients: effect of feedback to patients and their physicians: a randomized controlled trial. Diabetes Care 200124 690-694.

18 New JP, Mason JM, Freemantle N. Teasdale S, Wong LM, Bruce NJ, Burns JA \& Gibson JM. Specialist nurse-led intervention to treat and control hypertension and hyperlipidemia in diabetes (SPLINT): a randomized controlled trial. Diabetes Care 200326 2250-2255.

19 Davidson MB. Effect of nurse-directed diabetes care in a minority population. Diabetes Care 200326 2281-2287.

20 Denver EA, Barnard M, Woolfson RG \& Earle KA. Management of uncontrolled hypertension in a nurse-led clinic compared with conventional care for patients with type 2 diabetes. Diabetes Care $2003262256-2260$.
21 Glasgow RE, Funnell MM, Bonomi AE, Davis C, Beckham V \& Wagner EH. Self-management aspects of the improving chronic illness care breakthrough series: implementation with diabetes and heart failure teams. Annals of Behavioural Medicine 200224 $80-87$.

22 Marvel MK, Epstein RM, Flowers K \& Beckman HB. Soliciting the patient's agenda: have we improved? Journal of the American Medical Association 1999281 283-287.

23 Levinson W, Gorawara-Bhat R \& Lamb J. A study of patient clues and physician responses in primary care and surgical settings. Journal of the American Medical Association $2000 \quad \mathbf{2 8 4}$ $1021-1027$.

24 Dijkstra R, Braspenning J \& Grol R. Empowering patients: how to implement a diabetes passport in hospital care. Patient Education and Counselling 200247 173-177.

25 DAFNE Study Group, Training in flexible, intensive insulin management to enable dietary freedom in people with type 1 diabetes: dose adjustment for normal eating (DAFNE) randomised controlled trial. British Medical Journal 2002325 746-752.

26 Mühlhauser I, Jorgens V, Berger M, Graninger W, Gurtler W, Hornke L, Kunz A, Schernthane G, Scholz V \& Voss HE. Bicentric evaluation of a teaching and treatment programme for type 1 (insulin-dependent) diabetic patients: improvement of metabolic control and other measures of diabetes care for up to 22 months. Diabetologia 198325 470-476.

27 Anderson RM, Funnell MM, Nwankwo R, Gillard ML \& Fitzgerald JT. Evaluation of a problem-based culturally specific, patient education program for African-Americans with diabetes. Diabetes 200050 (Suppl 2) A195.

Received 19 May 2004

Accepted 24 May 2004 